\title{
Resolved debris disc emission around $\eta$ Telescopii: a young solar system or ongoing planet formation?
}

\author{
R. Smith ${ }^{1}$, L. J. Churcher ${ }^{1}$, M. C. Wyatt ${ }^{1}$, M. M. Moerchen ${ }^{2,3}$, and C. M. Telesco ${ }^{2}$ \\ ${ }^{1}$ Institute of Astronomy (IoA), University of Cambridge, Madingley Road, Cambridge, CB3 0HA, UK \\ e-mail: rsed@ast.cam.ac.uk \\ 2 Department of Astronomy, University of Florida, 211 Bryant Space Science Center, PO Box 112055, Gainesville, FL, \\ 32611-2055, USA \\ 3 European Southern Observatory, Alonso de Cordova 3107, Casilla 19001, Vitacura, Santiago 19, Chile
}

Received 29 July 2008 / Accepted 30 October 2008

ABSTRACT

\begin{abstract}
Aims. Sixty percent of the A star members of the 12 Myr old $\beta$ Pictoris moving group (BPMG) show significant excess emission in the mid-infrared, several million years after the proto-planetary disc is thought to disperse. Theoretical models suggest this peak may coincide with the formation of Pluto-sized planetesimals in the disc, stirring smaller bodies into collisional destruction. Here we present resolved mid-infrared imaging of the disc of $\eta \mathrm{Tel}$ (A0V in the BPMG) and consider its implications for the state of planet formation in this system.

Methods. The source was observed at 11.7 and $18.3 \mu \mathrm{m}$ using T-ReCS on Gemini South. The resulting images were compared to simple disc models to constrain the radial distribution of the emitting material.

Results. The emission observed at $18.3 \mu \mathrm{m}$ is shown to be significantly extended beyond the PSF along a position angle $8^{\circ}$. This is the first time dust emission has been resolved around $\eta$ Tel. Modelling indicates that the extension arises from an edge-on disc of radius $0.5 \operatorname{arcsec}(\sim 24 \mathrm{AU})$. Combining the spatial constraints from the imaging with those from the spectral energy distribution shows that $>50 \%$ of the $18 \mu \mathrm{m}$ emission comes from an unresolved dust component at $\sim 4 \mathrm{AU}$.

Conclusions. The radial structure of the $\eta$ Tel debris disc is reminiscent of the Solar System, suggesting that this is a young Solar System analogue. For an age of $12 \mathrm{Myr}$, both the radius and dust level of the extended cooler component are consistent with selfstirring models for a protoplanetary disc of 0.7 times minimum mass solar nebula. The origin of the hot dust component may arise in an asteroid belt undergoing collisional destruction or in massive collisions in ongoing terrestrial planet formation.
\end{abstract}

Key words. circumstellar matter - infrared: stars - planetary systems - stars: individual: $\eta$ Telescopii

\section{Introduction}

Debris discs around nearby stars are often discovered through the detection of emission beyond that predicted to come from the stellar photosphere in the infrared or sub-millimeter (see Wyatt 2008, for a recent review). The detected emission arises from the absorption and subsequent re-emission of starlight at longer wavelengths by dust particles in the discs. These dust grains are believed to be continuously reproduced by the collisional destruction and/or sublimation of larger bodies (planetesimals), as the lifetimes of such small grains due to removal by radiation pressure or Poynting-Robertson (P-R) drag are small compared to the age of the star (Backman \& Paresce 1993). Studying these debris discs can give clues about how the circumstellar region has evolved during the star's formation and subsequent lifetime, how any planetary system around the star may have formed and evolved, and the current status of the system (see e.g., Wyatt et al. 1999).

The star $\eta$ Tel (HD 181296) is an A0Vn member of the $\beta$ Pictoris moving group, with an age estimated to be $12_{-4}^{+8} \mathrm{Myr}$ (age estimated for moving group in Zuckerman et al. 2001). It has an M7/8V companion at an offset of $4^{\prime \prime}$ at a position angle of $160^{\circ}$ to the star (Lowrance et al. 2000). Hipparcos listed parallax measurements put this system at a distance of $48 \mathrm{pc}$, giving a projected offset of $192 \mathrm{AU}$ for the companion.
Backman \& Paresce (1993) first identified this star as a debris disc candidate based on IRAS measurements which indicate that excess emission is present at 12,25 and $60 \mu \mathrm{m}$. The excess measurement at $12 \mu \mathrm{m}$ is particularly rare (Aumann \& Probst 1991), as debris discs are more typically characterised by dust at several $10 \mathrm{~s}$ of $\mathrm{AU}$ which is too cool to emit at $12 \mu \mathrm{m}$. Indeed the majority of A star sources with disc emission resolved at thermal wavelengths have discs extended on $100 \mathrm{~s}$ of AU scales $(\beta$ Pic, HR4796A, 49 Ceti, HD 141596 and HD 32297, Backman \& Paresce 1993; Jayawardhana et al. 1998; Wahhaj et al. 2007; Fisher et al. 2000; Moerchen et al. 2007a). Nevertheless, examples of A stars with dust confirmed to lie within 10 AU are known (e.g., $\zeta$ Lep, Moerchen et al. 2007b), and there remains debate as to whether such hot emission can originate in the steady-state destruction of planetesimal belts (Wyatt et al. $2007 b$ ) or in collisions between growing protoplanets that are expected during terrestrial planet formation (Kenyon \& Bromley 2002). Chen et al. (2006) presented IRS observations of $\eta \mathrm{Tel}$ showing that the excess emission cannot be fit by a single temperature of dust as the spectrum is too flat. They interpret this as evidence for two temperatures of dust, but from the emission spectrum alone it is not possible to tell if this arose from dust in two separate radial locations around the star, or alternatively two different grain populations at the same radial location. The aim of this paper is to use resolved imaging of the disc to allow a 
differentiation between these alternatives. In addition these images will confirm that the excess emission is in fact centred on the primary star and not the binary, although due to the brightness of the $12 \mu \mathrm{m}$ flux it is unlikely that the emission arises from dust around the M7/8 companion.

The age of this system and membership of the moving group make it very interesting in terms of evolutionary studies of debris discs. Recent work by Currie et al. (2007) has shown that excess emission at $24 \mu \mathrm{m}$ around A stars increases from 5-10 Myr, peaks around 10-15 Myr and then declines with age. The cause of the peak is not yet known (see review in Wyatt 2008), but is suggested to be the delayed formation of Pluto-sized bodies further from the star, as these bodies are necessary to stir the disc so that collisions are destructive thus releasing large quantities of dust (Kenyon \& Bromley 2004). The 12 Myr $\beta$ Pictoris moving group has $5 \mathrm{~A}$ star members, 3 of which are known to have debris discs that exhibit excess emission in the mid-infrared $(\beta$ Pictoris, HD 172555 and $\eta$ Tel; Chen et al. 2006; Rebull et al. 2008), and the proximity of this group means it offers the chance to assess the origin and diversity of the peak.

Here we present 11.7 and $18.3 \mu \mathrm{m}$ imaging of $\eta$ Tel taken with the T-ReCS instrument on Gemini South which shows a resolved debris disc at $18.3 \mu \mathrm{m}$. We confront these observations with models to determine the optimal disc parameters and discuss the implications of the inferred structure for the status of planet formation in this system, both by comparison with the self-stirred models of Kenyon \& Bromley (2004) and with our understanding of the early Solar System.

\section{Observations}

The source was observed under proposal GS-2007A-Q-45 using T-ReCS on Gemini South with filter Qa $\left(\lambda_{\mathrm{c}}=18.3 \mu \mathrm{m}\right.$, $\Delta \lambda=1.51 \mu \mathrm{m})$ and $\mathrm{Si}-5\left(\lambda_{\mathrm{c}}=11.66 \mu \mathrm{m}, \Delta \lambda=2.13 \mu \mathrm{m}\right)$, which we refer to as $\mathrm{Qa}$ and $\mathrm{Si}-5$ observations henceforth. The pixel scale of the T-ReCS imager is 0. '09 with a total field of view of $29^{\prime \prime} \times 22^{\prime \prime}$. The observations were taken in parallel chopnod mode with a chop throw of $10^{\prime \prime}$ and chop position angle of $55^{\circ}$ (East of North) at Q, $100^{\circ}$ at N. The Qa observations were taken over two consecutive nights (1st and 2nd July 2007), with the Si-5 observations taken on one night only (12th July 2007). Total integration time was $9120 \mathrm{~s} \mathrm{(4560} \mathrm{s} \mathrm{on} \mathrm{source)} \mathrm{at} \mathrm{Qa,} \mathrm{and}$ $912 \mathrm{~s}$ (456 s on source) at Si-5. Observations of standard stars HD 196171 (spectral type K0III) and HD 179886 (spectral type K3III), both listed in Cohen et al. (1999), were made to calibrate the photometry and also determine the PSF of the observations. The order of observations and integration times are shown in Table 1.

These data for $\eta$ Tel were taken in multiples of 304 s-long integrations, each consisting of 7 complete nod cycles (ABBA). The data were reduced using custom routines described in Smith et al. (2008). Data reduction involved determination of a gain map using the mean values of each frame to determine pixel responsivity (masking off pixels on which emission from the source could fall, equivalent to a sky flat). In addition a dcoffset was determined by calculating the mean pixel values in columns and rows (excluding pixels on which source emission was detected) and this was subtracted from the final image to ensure a flat background. Pixels showing high or low gain, or those which showed great variation throughout the observation were masked off. In order to avoid errors in co-adding the data which could arise from incorrect alignment of the images, we used re-binned images in which each pixel was a fifth the size of the T-ReCS image pixels to determine an accurate centre of the
Table 1. Observations in order and total integration times of the observations taken under proposal GS-2007A-Q-45.

\begin{tabular}{ccccc}
\hline \hline Date & Object & Filter & $\begin{array}{c}\text { Integration } \\
\text { time (s) }\end{array}$ & $\begin{array}{c}\text { Calibrated } \\
\text { flux, mJy }\end{array}$ \\
\hline $01 / 07 / 2007$ & HD 196171 & Qa & 152 & 6058 \\
$01 / 07 / 2007$ & $\eta$ Tel & Qa & 1824 & $350 \pm 11$ \\
$01 / 07 / 2007$ & HD 196171 & Qa & 152 & 6058 \\
$01 / 07 / 2007$ & $\eta$ Tel & Qa & 1824 & $347 \pm 11$ \\
$01 / 07 / 2007$ & HD 196171 & Qa & 152 & 6058 \\
\hline $02 / 07 / 2007$ & HD 196171 & Qa & 152 & 6058 \\
$02 / 07 / 2007$ & $\eta$ Tel & Qa & 1824 & $345 \pm 10$ \\
$02 / 07 / 2007$ & HD 196171 & Qa & 152 & 6058 \\
$02 / 07 / 2007$ & $\eta$ Tel & Qa & 1824 & $350 \pm 11$ \\
$02 / 07 / 2007$ & HD 196171 & Qa & 152 & 6058 \\
$02 / 07 / 2007$ & $\eta$ Tel & Qa & 1824 & $331 \pm 10$ \\
$02 / 07 / 2007$ & HD 196171 & Qa & 152 & 6058 \\
\hline $12 / 07 / 2007$ & HD 179886 & Si-5 & 304 & 4417 \\
$12 / 07 / 2007$ & $\eta$ Tel & Si-5 & 912 & $342 \pm 18$ \\
$12 / 07 / 2007$ & HD 179886 & Si-5 & 304 & 4417 \\
\hline
\end{tabular}

Note that on-source integration time is half the total integration time listed in the table.

stellar images, aligned and co-added these images and then rebinned to the original pixel size. The re-binning was performed using bilinear interpolation across the array. This technique was used both to determine a final image of $\eta$ Tel and standard star for each band. To ensure that background noise did not affect the re-binning process the same procedure was applied with an additional step of smoothing the images with a Gaussian kernel of $F W H M$ equal to that of the standard star image before rebinning. It was found that the relative sizes of the final images of $\eta \mathrm{Tel}$ and the standard stars and ellipticity of the images was unchanged by this additional step, indicating that the re-binning was not affected by background noise. This is as to be expected in data such as this where the detector super-samples the PSF.

\section{Results}

The final co-added images of $\eta \mathrm{Tel}$ in both filters and the standard star for PSF reference are shown in Figure 1. The contours shown in the images are at $20 \%, 40 \%, 60 \%$ and $80 \%$ of the peak in each case (peak value in Qa $5.24 \mathrm{mJy} / \mathrm{pixel}$, in Si-5 $9.89 \mathrm{mJy} / \mathrm{pixel}$ ). Also shown in the right-hand image for each row is the residual emission around $\eta$ Tel observed when subtracting the standard star image as a PSF reference scaled to the peak of the $\eta$ Tel image. The contours and residuals are discussed further below.

Photometry was performed using 1'.0 radius circular apertures centred on the stellar image. The centre of the image was determined through a 2-dimensional Gaussian fit. An average of the calibration factors determined from the standard star observations taken on the night was used for corresponding photometric calibration. Statistical noise was determined using an annulus with inner radius $1^{\prime \prime}$ and outer radius $2^{\prime \prime}$ centred on the source, resulting in uncertainties of 0.13 and $0.06 \mathrm{mJy} / \mathrm{pixel}$ at Qa and Si-5 respectively. The calibration factors were found to vary by $4 \%$ and $2 \%$ on the first and second nights of Qa band observations, respectively, which is consistent with the variation of $3 \%$ found between fluxes measured on individual $304 \mathrm{~s}$ integrations on $\eta \mathrm{Tel}$ (which is bright enough for an $\sim 60$ sigma detection even in such a short integration). Although the calibration 
$\eta \mathrm{Tel}$
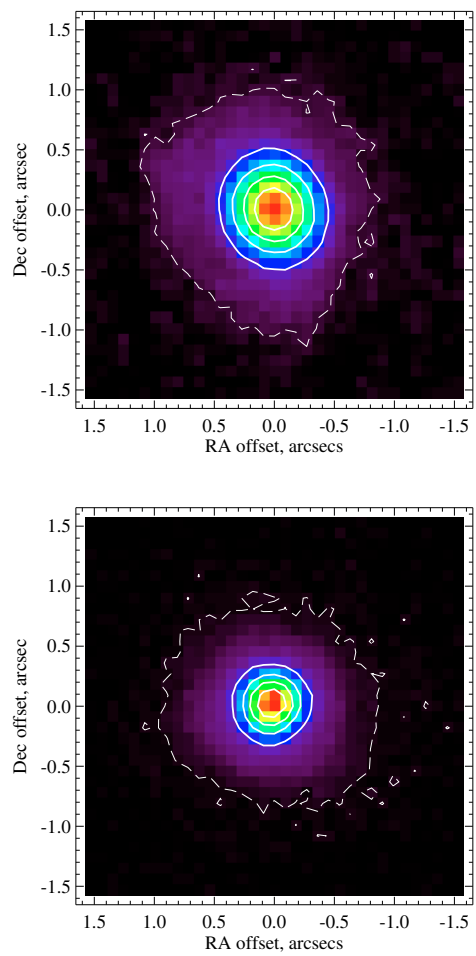

Standard
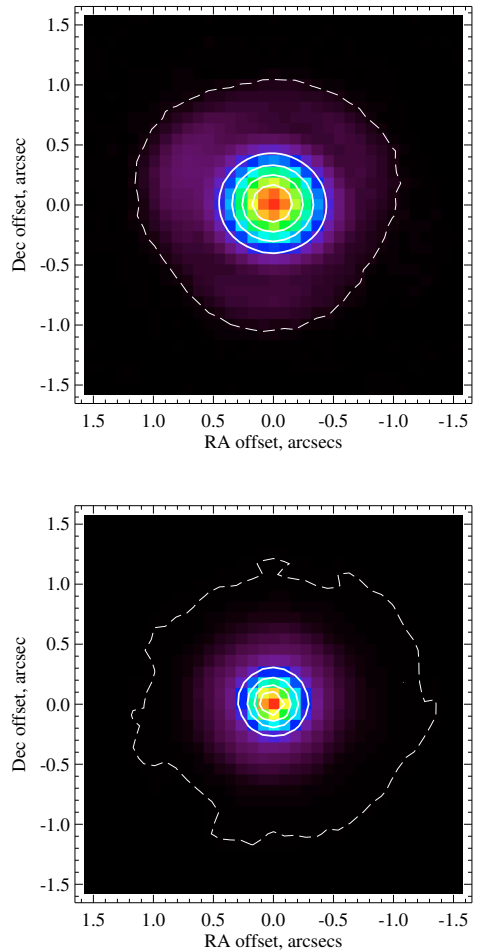

Residual
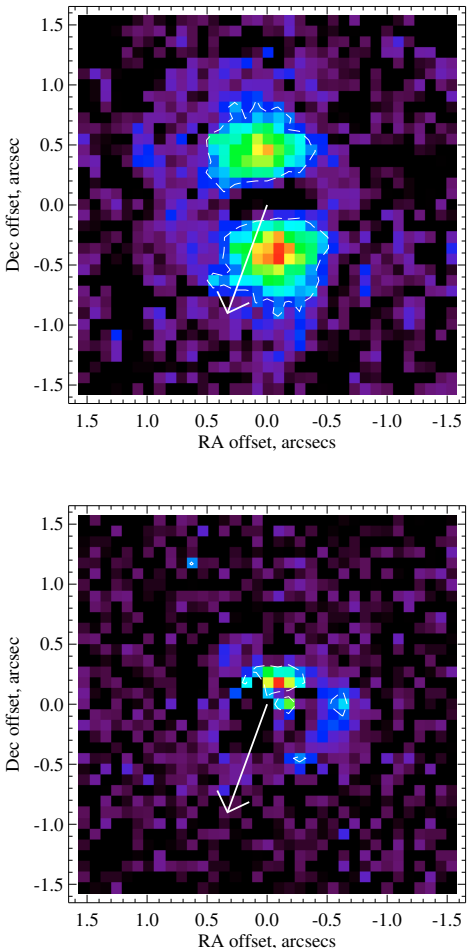

Fig. 1. The co-added final images from the Qa observations (top row) and the Si-5 observations (bottom). Orientation of the images is North up, East left. Left: the total co-added images of $\eta$ Tel. Middle: the co-added images of the standard star forming our reference PSF. Note the distinct ellipticity with major axis in the North-South direction seen in the Qa image of $\eta$ Tel is not seen in Si-5 or in the standard star images. Solid line contours are at the 20\%, 40\%,60\%, and 80\% of the peak levels for all images. Peaks are at a level of $650 \mathrm{mJy} / \mathrm{arcsec}^{2}$ for $\eta \mathrm{Tel}$ at Qa, $1220 \mathrm{mJy} / \operatorname{arcsec}^{2}$ for $\eta \mathrm{Tel}$ at Si-5, $13990 \mathrm{mJy} / \operatorname{arcsec}^{2}$ for the standard star at Qa and $20670 \mathrm{mJy} / \operatorname{arcsec}^{2}$ for the standard at Si-5. Right: the residual emission after subtraction of the total standard star image scaled to the peak of the image of $\eta$ Tel. The dashed contour indicates the $3 \sigma$ per pixel level (from background noise). The colour scale is linear from 0 to the maximum pixel value in each image. The residual images have peak brightness of $88 \mathrm{mJy} / \mathrm{arcsec}^{2}$ at Qa and $35 \mathrm{mJy} / \mathrm{arcsec}^{2}$ at Si-5. The arrow indicates the direction of the M7/8V binary which lies at $4^{\prime \prime}, \mathrm{PA}{ }^{\circ} 160$. Note that the emission seen in the Si-5 residual image is not significant $(<1 \sigma$ significance) when the variation in the PSF is considered.

factors varied by just 5\% for the two observations of standards in the Si-5 filter, analysis of the individual $304 \mathrm{~s}$ integrations on $\eta$ $\mathrm{Tel}$ (each of which resulted in a 78 sigma detection) showed that photometric accuracy was in fact at a level of $13 \%$. Our photometry yields total fluxes of $342 \pm 44 \mathrm{mJy}$ in $\mathrm{Si}-5$ (with $\mathrm{S} / \mathrm{N}$ of 292 ) and $345 \pm 15 \mathrm{mJy}$ in Qa (with $\mathrm{S} / \mathrm{N}$ of 129 ), including both calibration and statistical noise, where the $\mathrm{S} / \mathrm{N}$ given in brackets indicates the level of statistical noise. Standard stars were observed at a similar airmass to $\eta$ Tel. In addition, calibration levels were compared to airmass for the standard star observations and no correlation was found, therefore no correction for extinction was applied to calibration. Using a Kurucz model profile scaled to the 2MASS K band magnitude, the stellar flux in filters Si-5 and Qa is expected to be $282 \mathrm{mJy}$ and $114 \mathrm{mJy}$, respectively. Thus the photometry gives an excess of $60 \pm 44 \mathrm{mJy}$ and $231 \pm 15 \mathrm{mJy}$ in Si-5 and Qa. The IRS spectrum of Chen et al. (2006) after subtraction of the above mentioned photospheric emission gives excess emission of $150 \pm 5$ and $257 \pm 2 \mathrm{mJy}$, respectively, at these two wavelengths and so the photometry presented here is in agreement within the T-ReCS photometric errors with the IRS results at the $2 \sigma$ level. Thus it does not appear that the IRS spectrum includes any emission centred on the star outside the $1^{\prime \prime}$ aperture used here for photometry, as might happen in the larger IRS beam (extracted along a 3".7 slit).

Figure 1 shows the final co-added images of $\eta$ Tel and the standard star for a PSF reference. The top line of this figure shows the Qa total image of $\eta$ Tel and the standard. An elliptical shape can clearly be discerned, and a 2-dimensional Gaussian fit to the image has an ellipticity of $0.117 \pm 0.007$ with the major axis at $19.9 \pm 2.0^{\circ}$ East of North; the same ellipticity was seen on the two separate nights of Qa observing. For comparison, the ellipticity of the final standard image is $0.084 \pm 0.005$ with the major axis at $83 \pm 2^{\circ}$. This slightly elliptical shape of the PSF is seen across the standard star integrations (see later this section) and does not coincide with the extension seen in the $\eta$ Tel images. As all Qa observations were performed with no onsky rotation and chop-nod performed at $55^{\circ}$, the direction of the extension is unlikely to be the result of chop smearing.

Also shown in Fig. 1 is the residual emission of $\eta$ Tel following a subtraction of the PSF reference standard star image scaled to the peak of the $\eta$ Tel image, which subtracts a total of $252 \mathrm{mJy}$ from the image. Although this subtraction method is likely to remove some of the disc emission (some of which contributes to the peak surface brightness), it does highlight the location of the non-pointlike emission. Peaks are seen in the Qa residual image that are centred at $0{ }^{\prime} 45$ (21.6 AU), 5.2 ${ }^{\circ}$ East of North, and $0 .^{\prime} 40$ (19.2 AU), $190.5^{\circ}$ East of North. Symmetrical peaks in the residual emission either side of the star are exactly what would be expected for an edge-on ring (Telesco et al. 2000, although also note that we cannot rule out a less inclined ring without detailed modelling of the disc, see Sect. 4). Since the residual peaks are more representative of the extension seen than the 2-dimensional 

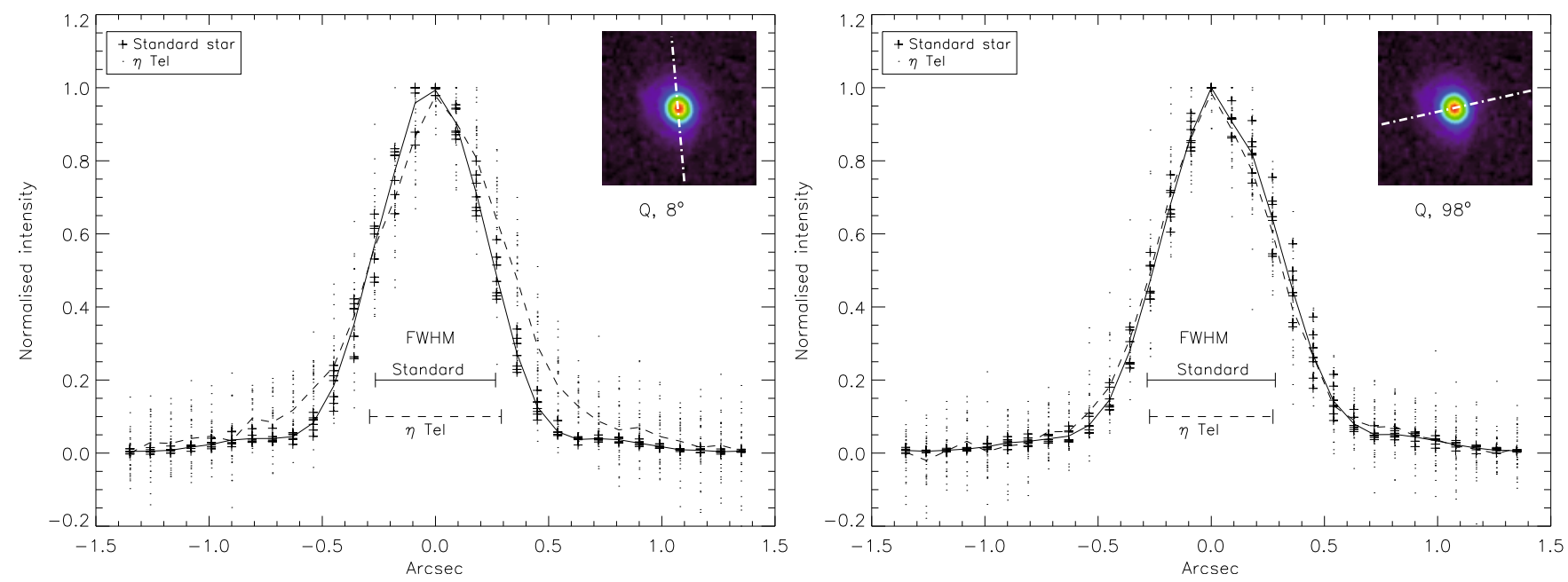

Fig. 2. The profiles of line cuts through the total images, at $8^{\circ}($ left $)$ and $98^{\circ}$ (right) at Qa. The median $F W H M$ measurements taken over all sub-integrations from Gaussian fits to the profiles at the two angles are indicated for reference. Also shown are averaged profiles from all the line cuts for both $\eta$ Tel and the standard star. Observe that the profile for $\eta$ Tel at $8^{\circ}$ is much broader in the wings than the standard star. Note that multiple intensity profiles for the science target and standard are shown here. These are taken from longer integrations than noted in the text to reduce the number of profiles and improve the clarity of the figure.

Gaussian fit discussed above, hereafter the extension is considered to be at a position angle of $\sim 8^{\circ}$. Thus the extension is at $\sim 28^{\circ}$ with respect to the direction of the binary (shown with an arrow), suggesting if this arises from an edge-on disc, then that disc is not in the same plane as the binary. The flux in the residual peaks totals $80 \pm 5 \mathrm{mJy}$ (measured in 0.4 radius apertures; the error term includes background and calibration uncertainty added in quadrature), which as expected is below that of the excess emission $231 \pm 15$ mJy measured in our photometry. An estimate of the level of disc emission which may have been removed from the residuals image by our method of PSF subtraction can be determined from consideration of how much the two peaks may have contributed to the central pixel. Using the PSF model scaled to the residual peaks this was calculated as $0.3 \mathrm{mJy}$ (as compared to the total peak before subtraction of $5.24 \mathrm{mJy}$ ). This method suggests the extended emission represents a minimum of $6 \%$ of the peak emission, as if the extended emission is truly a disc lying across the star, this method will again underestimate the disc flux in the central pixel. A more detailed model of the extended emission seen in the Qa image is discussed in Sect. 4.

To assess the temporal variability of this $F W H M$ and the significance of the extensions, as well as to search for a dependence on time or airmass, linecuts were performed at position angles of $8^{\circ}$ and $98^{\circ}$ for individual nod set images $(22 \mathrm{~s})$ for the standard star and two nod set images (43 s) for $\eta$ Tel. Two methods of fitting the cuts, by a Gaussian profile and a Moffat profile were tried. Both fits provided the same relative results, although the peak was normally fitted more accurately using the Gaussian profile. No dependence on airmass or time was found, and median $F W H M$ values and standard errors were found to be: for the standard target $0.531 \pm 0 .{ }^{\prime} 002$ at $8^{\circ}, 0.0^{\prime} 566 \pm 0 .^{\prime} 004$ at $98^{\circ}$; and for $\eta$ Tel $0.582 \pm 00^{\prime} 010$ at $8^{\circ}$ and $0 . ' 545 \pm 0.007$ at $98^{\circ}$. The lower signal-to-noise on the $\eta \mathrm{Tel}$ observations naturally leads to a larger degree of scatter in the intensity profiles. However it is clear that at Qa there is no difference in the $F W H M$ of the $\eta$ Tel frames and standard star target frames at $98^{\circ}$, but that at $8^{\circ}$ the $F W H M$ is larger for $\eta$ Tel than for any of the individual integrations of the standard stars. A further graphical demonstration of the significance of the extension is provided by Fig. 2 which shows the cuts through each Qa integration (of 304 s) from the standard star imaging (plus signs) and the $\eta$ Tel observations (dots). At $8^{\circ}$ the $\eta$ Tel sub-integrations are not only broader at the FWHM but also have broader wings to their profiles compared to the standard star images. Neither of these features is seen at $98^{\circ}$.

The Si-5 image of $\eta$ Tel shown in Fig. 1 includes only the first 2 of 3 integrations on the source. The final integration showed an unusual shape compared to the previous integrations: a greater extension in the North-South direction, and an unusual asymmetric shape, with an extension towards the East of the image not matched on the West side. This shape was seen in all but the first nod set. Neither the North-South extension nor the Easterly extension match the chop PA of $55^{\circ}$. The eccentricities and orientations of the major axis for the 3 integrations are: $0.027 \pm 0.009$ at $110^{\circ} ; 0.082 \pm 0.009$ at $167^{\circ}$; and $0.163 \pm 0.012$ at $80^{\circ}$. Thus we consider that the shape seen in the final integration is not evidence for true extension, but is due to the variable seeing conditions on this night.

In the Si-5 residual image we see only low significance emission at the Northern edge of the original stellar image. The emission seen in this residual image (Fig. 1 lower right image) is $6.6 \pm 0.9 \mathrm{mJy}$ (this error includes only background error, not PSF uncertainty), however with subtraction of individual standard star images rather than the coadded standard star PSF reference image flux in this region can be as low as $-7.8 \mathrm{mJy}$, demonstrating the importance of consideration of the PSF variation when determining evidence of extended emission close to the star, and the true insignificance of this emission. Again the linecuts for individual nodsets, both for $\eta$ Tel and standard, were assessed. No dependence on airmass or time was found, and median values and standard errors of the Gaussian FWHM measurements excluding the final integration for $\eta$ Tel (see above) are: for the standard target $0.383 \pm 0 .^{\prime} 003$ at $8^{\circ}, 0.407 \pm 00^{\prime} 006$ at $98^{\circ}$; and for $\eta$ Tel $0 . ' 386 \pm 0.003$ at $8^{\circ}$ and $0 .^{\prime} 414 \pm 0 . ' 013$ at $98^{\circ}$. Thus examination of the PSF uncertainty indicates that the residual emission is likely to be the result of PSF variation over the course of the observation. 

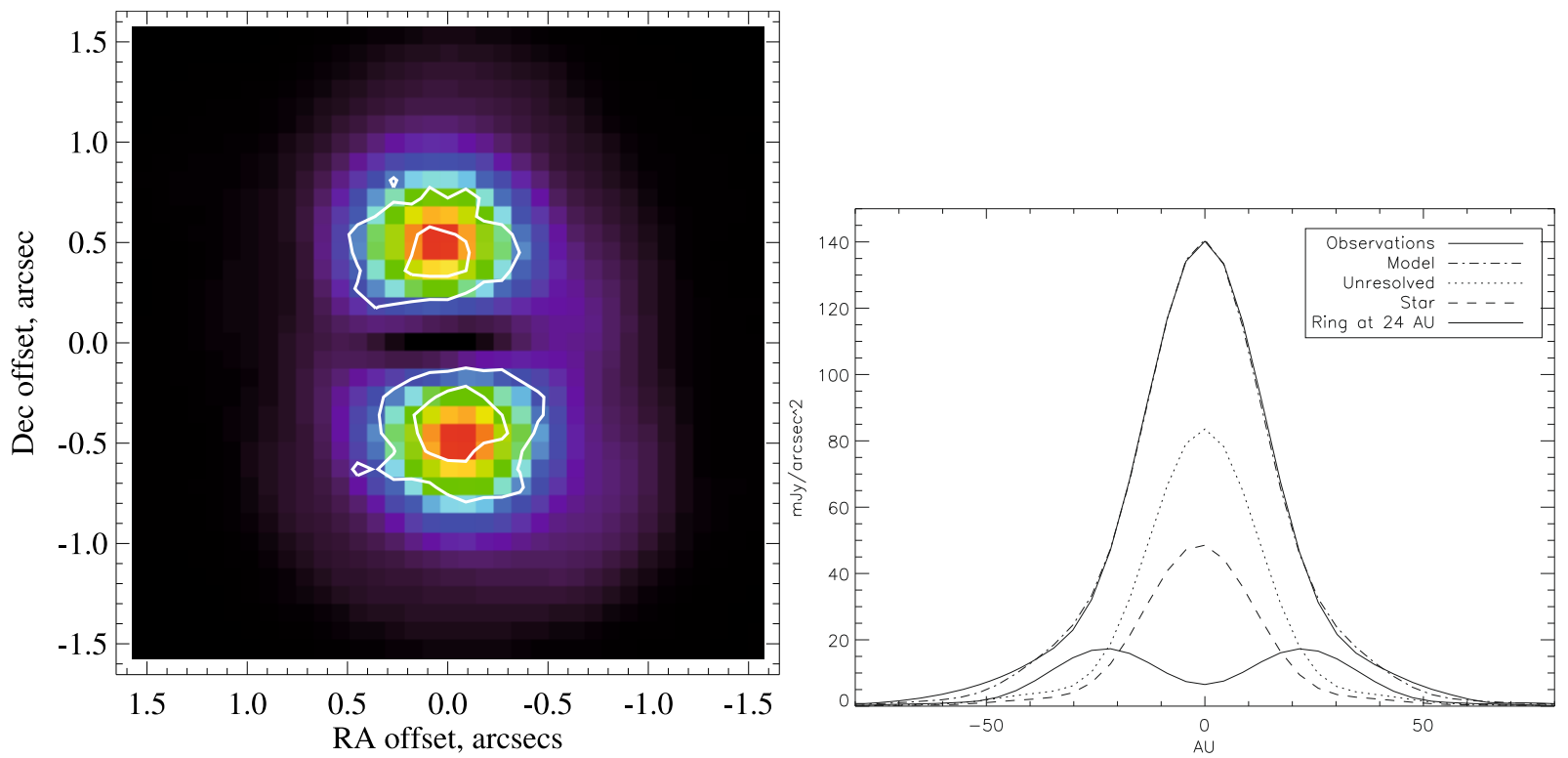

Fig. 3. The best fitting two parameter model for the Qa band emission around $\eta$ Tel. This model includes that stellar flux, an unresolved flux component at $205 \mathrm{mJy}$ and and an edge-on resolved disc component between 21-26 AU. Left: the model "residual" image, with contours from the observed residuals image (Fig. 1 top right). The model was convolved with the PSF and subjected to the same subtraction (standard star PSF reference image scaled to peak) as the $\eta$ Tel image. The scale is the same as the observed residuals image. The observed residuals contours show the good correspondence of the model with the observed emission and are at $1 / 3$ and $2 / 3$ of the residual peak. Right: the line cut through the $\eta$ Tel Qa image at $8^{\circ}$ and the best fitting model at the same position angle.

\section{Modelling the emission}

In order to determine the constraints on the radial distribution of emission provided by the resolved Qa imaging, we considered models for disc structure of increasing complexity. For all models it is assumed that the images are comprised of unresolved stellar flux (of $114 \mathrm{mJy}$ ), plus an additional unresolved disc component (motivated by the hot component inferred by Chen et al. 2006) of flux $F_{\text {unres }}$, along with an additional resolved disc component of flux $F_{\text {res }}$. The IRS photometry was used to constrain the total flux so that $F_{\text {unres }}+F_{\text {res }}=257$ mJy. The resolved flux is assumed to arise from an axisymmetric disc of opening angle $5^{\circ}$ (which is not constrained by the modelling) which lies at an inclination $I$ to our line-of-sight. Since the emission is consistent with an edge-on disc $I=0$ is assumed in the modelling, although the extent to which it is edge-on is constrained later (see Sect. 4.2). Two types of resolved disc were assumed. Narrow rings are defined by $r$, the radius of the mid-point of the disc, in AU; a finite ring width of $\mathrm{d} r / r=0.2$ was assumed, with a constant surface density. Extended rings were defined by a continuous distribution of surface density from some inner radius $r_{\text {in }}$ to an outer radius $r_{\text {out }}$ with surface density following a powerlaw $\Sigma \propto r^{\gamma}$, assuming grains with black body temperatures and emissivities.

The total models (discs and point sources) were convolved with the average PSF (shown in Fig. 1) for comparison with the observed data. The compatibility of each model with the observed structure was tested by taking line cuts along the direction of extension (at a PA of $8^{\circ}$ ). The line cuts were averaged over 3

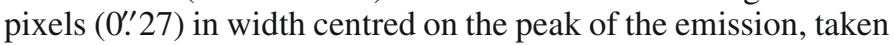
over a length of 50 pixels (i.e., \pm 2 '" 25 from the centre) to give a 1 dimensional profile for both the observation of $\eta$ Tel (with error bars) and for each model tested (e.g., Fig. 3, right). For each model described below a range of parameters was tried and a $\chi^{2}$ goodness-of-fit test applied to find the best fitting parameters and confidence limits (errors from background statistical error).
In addition to the $\chi^{2}$ from the line-cuts, that from a comparison of the model images and observation was also considered (e.g., Fig. 3, left).

To test whether more complicated models are necessary to explain the images, a Bayesian Information Criterion (BIC) test was applied to the model fitting:

$B I C=N \ln \left(\chi_{\min }^{2}\right)+k \ln N$,

where $N$ is the number of data points and $k$ the number of free parameters, and $\chi_{\min }^{2}$ is the minimum $\chi^{2}$ value found from each model (Schwarz 1978; see also Liddle 2004; Wahhaj et al. 2005). This test penalises models for having unnecessary parameters. A lower value of the BIC is preferred; a difference of 2 between BICs of different models indicates positive evidence against the higher BIC value, and a difference of 6 indicates strong evidence against the higher BIC value.

\subsection{One parameter models}

The simplest model is one with no resolved disc component. In this case we allowed $F_{\text {unres }}$ to be a free parameter, but as expected from the images and comparison of radial profiles in Fig. 2, the $\chi^{2}$ testing showed that no model provided a good fit to the profile with $\chi_{\min }^{2}=786$ and a reduced $\chi_{\mathrm{r}}^{2}=16$, where $\chi_{\mathrm{r}}^{2}=1.0$ is a perfect fit. The BIC of this model was 98 (from the 1 dimensional profiles).

Another possibility is to assume that there is no unresolved disc component, just a narrow ring defined by the free parameter $r$. Values of $r$ tested in this case were in the range 8-60 AU. This lower limit was chosen as smaller discs appear unresolved and thus provide the same goodness-of-fit as the unresolved emission only model described above. In this case the fit to the observed profile was poor, worse than assuming the emission to be completely unresolved with $\chi_{\min }^{2}=2815$ and a reduced $\chi_{\mathrm{r}}^{2}=59$. The BIC of this model was 160 . This demonstrates that the images 
confirm the suggestion of Chen et al. that the material is at multiple radii.

\subsection{Two parameter (preferred) model}

The two free parameter model assumes that the disc emission is comprised of a narrow ring of radius $r$ and an unresolved component at a level $F_{\text {unres }}$ (and so is essentially a combination of the two one parameter models considered above). The free parameter $F_{\text {unres }}$, which also controls the value of $F_{\text {res }}$, was varied between 0 and $280 \mathrm{mJy}$ (in $35 \mathrm{mJy}$ steps), while $r$ was tested with values between 0 and $95 \mathrm{AU}$ (in $3 \mathrm{AU}$ intervals). A much better fit was achieved with the two parameter model with an unreduced $\chi_{\min }^{2}=53$ and a reduced $\chi_{\text {rmin }}^{2}=1.13$ for $r=24 \mathrm{AU}$ and $F_{\text {unres }}=205 \mathrm{mJy}$ (corresponding to $F_{\text {res }}=54 \mathrm{mJy}$ ). The profile of this best fitting model is shown in Fig. 3 in which the contributions of the different components are identified. The BIC for this model was 31 (from 1 dimensional profiles) indicating that this is strongly favoured over the 1 parameter models.

The $\chi^{2}$ minimisation showed that the parameters were constrained to the ranges: $F_{\text {unres }}=116-225,136-231,96-243 \mathrm{mJy}$ and $r=16-32,10-42,10-53 \mathrm{AU}$ at the 1,2 , and $3 \sigma$ levels respectively (ranges from a marginalisation of the joint distribution of the two parameters). Using the line cuts perpendicular to the direction of maximum extension the inclination to our line of sight was constrained to be less than $20^{\circ}$, since at $I \geq 20^{\circ}$ the model FWHM (0'.574) exceeds the observed FWHM (0'566 \pm $0{ }^{\prime} 004$ ) by more than $1 \sigma$.

Given the importance of PSF variation in the residual structure seen in the $\mathrm{N}$ band images, and as we used the average PSF in the modelling outlined above, we performed the same modelling process using the different PSFs measured in the single standard star observations (i.e. not co-added). The sensitivity of our best fit parameters on the chosen PSF was minimal, with typical $1 \sigma$ confidence regions close to those given in Sect. 4.2 (widest ranges were $8 \mathrm{AU}<r<32 \mathrm{AU}$ and $20 \mathrm{mJy}<$ $F_{\text {unres }}<339 \mathrm{mJy}$ ). Thus the variation in the observed PSF does not strongly change the best fitting model for the extended emission.

The final total model at Qa is shown after subtraction of the PSF scaled to the peak of the observed image of $\eta$ Tel in Fig. 3 along with the profile of this best fitting model with an edgeon disc (inclination $=0$ ). Subtracting the model image from the observation reveals that two are a close match within the statistical noise over the area of the image $\left(900\right.$ pixels $=22^{\prime \prime} 7 \times 22^{\prime \prime} 7$, reduced $\chi^{2}=1.3$ ).

\subsection{More complicated models}

As soon as the ring is assumed to be broad the number of free parameters increases to 3 or 4 , depending on whether an unresolved component was included: i.e., $r_{\text {in }}, r_{\text {out }}, \gamma$, and possibly $F_{\text {unres. }}$. We started by assessing whether the radial distribution could be explained by a single continuous distribution of dust, without an unresolved component. We tried model grids with $r_{\text {in }} \in(0.2,6) \mathrm{AU}$ spaced at $0.2 \mathrm{AU}$ intervals, $r_{\text {out }} \in(6,60) \mathrm{AU}$ in $1.5 \mathrm{AU}$ intervals and $\gamma$ tested at intervals of 0.5 with $\gamma \in$ $(-2,+2)$. This resulted in a poor fit to the data $\left(\chi^{2}=198\right.$ over the 1 dimensional profile, reduced $\chi^{2}=4.2$ ), and the BIC of 83 which suggests that the radial distribution is discontinuous.

It was possible to find a better fit to the observations than the two parameter model of Sect. 4.2 when $F_{\text {unres }}$ was also included as a free parameter; a reduced $\chi^{2}$ of 1.03 (from the $1 \mathrm{~d}$ profile) was found for $r_{\text {in }}=1.5_{-0.5}^{+2} \mathrm{AU}, r_{\text {out }}=45 \pm 5 \mathrm{AU}, \gamma=-0.5$ and $F_{\text {unres }}=170 \pm 26 \mathrm{mJy}$. However, the BIC calculation results in a value of 55 suggesting that the additional parameters required for an extended dust distribution are not justified to fit the data.

\subsection{Combining imaging and SED constraints}

The emission spectrum of $\eta$ Tel is shown in Fig. 4. Here the stellar photospheric emission has been modelled using a Kurucz atmosphere of $9506 \mathrm{~K}$ that has been scaled to the 2MASS K band photometry (implying a stellar luminosity of $22 L_{\odot}$ for $48 \mathrm{pc}$ ). The photosphere of the M 7.5 star companion is shown for reference and is modelled using $2700 \mathrm{~K}$ NEXTGEN model atmosphere (Hauschildt et al. 1999). This companion is outside the aperture used to determine the TReCS photometry. In any case the emission from this M 7.5 star at the wavelength range covered by both TReCS and the Spitzer IRS spectrum is below the background statistical noise level and thus it does not contribute to the photometry shown at $>5 \mu \mathrm{m}$. Photospheric contributions have been subtracted from the fluxes shown at $>5 \mu \mathrm{m}$ which represent the excess emission from the disc. The results of our imaging require that the emitting dust be at two radii, in agreement with the conclusions of Chen et al. (2006). These separate components are shown by open symbols in Fig. 4.

Modelling was performed to provide a two component fit to the SED in which each component (the unresolved excess and resolved disc) is pegged to its $18 \mu \mathrm{m}$ flux, with the unresolved component dominating the IRS spectrum $<18 \mu \mathrm{m}$ and the resolved component dominating the longer wavelength fluxes. We assume a maximum grain size of $1000 \mu \mathrm{m}$, with a minimum grain size of $10.8 \mu \mathrm{m}$ as this is the size at which grains are removed from the system by radiation pressure. Large grains (greater than $\mathrm{mm}$-sized) are inefficient absorbers and emitters of radiation, and their addition to the grain population would not have a great affect on the SED. A size distribution of $n(D) \propto$ $D^{-3.5}$ (see Dohnanyi 1969) was also assumed. We consider a distribution of grain sizes to be a realistic model for a dust population created in the different origin scenarios presented in Sect. 5 (Chen et al. 2006, use a single grain size to fit the IRS spectrum). Grains were assumed to have a silicate core and organic refractory/icy mantle (as used in Li \& Greenberg 1998; Augereau et al. 1999). Dielectric constants were calculated using Maxwell-Garnett effective medium theory and optical properties calculated using Mie theory, Rayleigh-Gans theory and Geometric Optics in the appropriate size regimes (Bohren \& Huffman 1983). The unresolved component was then best fit by a silicate fraction of $30 \%$ by volume (with the remaining volume composed of organic refractory material) and porosity of 0.2 , which fit the shape and level of the silicate feature at $\sim 10 \mu \mathrm{m}$, resulting in a dust population at $3.9 \mathrm{AU}$ of fractional luminosity $L_{\text {dust }} / L_{\star}=1.57 \times 10^{-4}$. Although we do not consider this modelling to have uniquely constrained the grain composition, this is a physically based model from which it is possible to estimate the radial location of the dust. Since this location is smaller than the FWHM it is confirmed that this should appear unresolved in our images, which would be expected as long as the radius was $\leq 6 \mathrm{AU}$.

The same dust grain size distribution was used to model the cold resolved component. The same dust composition with the dust located between 21 and $26 \mathrm{AU}$ as constrained by modelling the resolved Qa emission provides a good fit to the Spitzer IRS spectrum of the excess emission (Fig. 4). The fractional luminosity of the resolved component is similar to the unresolved component at $L_{\text {dust }} / L_{\star}=1.39 \times 10^{-4}$. The $\mathrm{N}$ band flux of the 


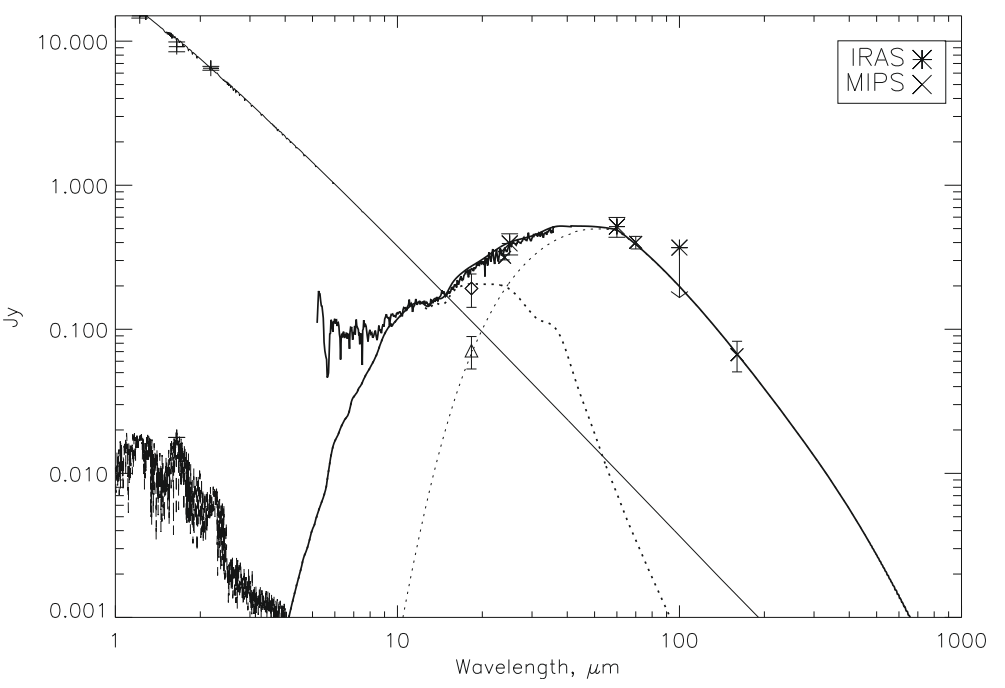

Fig. 4. Spectral energy distribution (SED) of $\eta$ Tel. The photosphere of $\eta$ Tel is fitted with a Kurucz model profile scaled to the 2MASS K band flux and shown with a solid line, as is the photosphere of the binary companion which is modelled with a NextGen model atmosphere (appears on this plot only shortwards of $5 \mu \mathrm{m}$ ). Observations at $>5 \mu \mathrm{m}$ are shown after the subtraction of photospheric contributions: the IRS spectrum of Chen et al. (2006) (solid line between 5-30 $\mu \mathrm{m}$ ), MIPS fluxes (Rebull et al. 2008, crosses), IRAS colourcorrected excess (asterisks) and the contribution to the $18 \mu \mathrm{m}$ fluxes from the unresolved (open triangle) and resolved (open diamond) components, as inferred from the modelling of Sect. 4.4 (see also Table 2). Note that the IRS excess emission spectrum at $\lesssim 8 \mu \mathrm{m}$ is subject to large uncertainty due to uncertainties in the level of photospheric emission in this range. The two component fit to the excess spectrum is shown by dotted lines - the hotter unresolved component at 3.9 AU and a resolved component at $24 \mathrm{AU}$.

Table 2. The observed emission from the T-ReCS observations of $\eta$ Tel, with comparison to the predicted emission from the two components used to fit the excess SED as described in Sect. 4.4.

\begin{tabular}{cccccc}
\hline \hline Band & $\begin{array}{c}\text { Observed } \\
\text { emission, mJy }\end{array}$ & $\begin{array}{c}\text { Photospheric } \\
\text { emission, mJy }\end{array}$ & $\begin{array}{c}\text { Observed Excess } \\
\text { emission, mJy }\end{array}$ & $\begin{array}{c}\text { Expected from } \\
\text { unresolved } \\
\text { component, mJy }\end{array}$ & $\begin{array}{c}\text { Expected from } \\
\text { resolved } \\
\text { component, mJy }\end{array}$ \\
\hline Si-5 & $342 \pm 44$ & 282 & $60 \pm 44^{a}$ & 140 & 6 \\
$\mathrm{Qa}$ & $345 \pm 15$ & 114 & $231 \pm 15$ & 185 & 62 \\
\hline
\end{tabular}

${ }^{a}$ Recall that the Si5 photometry suffered from poor calibration accuracy, and as such SED modelling was primarily fitted to the IRS spectrum at shorter wavelengths. Figure 4 shows the fit recreates the IRS spectrum accurately.

resolved component is just $6 \mathrm{mJy}$, which is consistent with the non-detection of the resolved emission in the imaging which is dominated by the unresolved component (at $140 \mathrm{mJy}$ ). The full SED fit was compared to the observed photometry and a $\chi^{2}$ goodness-of-fit computed. Data points from IRAS, MIPS, TReCS and the IRS spectrum were weighted according to their uncertainty. Data points at $<7 \mu \mathrm{m}$ were excluded from the fit due to high levels of uncertainty arising from the correct determination of the stellar contribution at this range. The resulting reduced $\chi^{2}$ of 1.14 indicates the model fits the observed emission well.

Thus the two component model provides a consistent picture of this disc (see Table 2 and Fig. 4 for comparison between model and observed emission levels), and while we cannot accurately assess the range of temperatures (and radii) that are present in the two components, the poor fit of a continuous surface density distribution (Sect. 4.3) suggests that there is a region of lower surface density between the two.

\section{Discussion}

In the following section we explore the possible origin of the two dust populations around $\eta$ Tel. We consider two possibilities: that $\eta$ Tel represents an analogue of the Solar System when it was at a similar age; and that the observed emission could arise from ongoing planet formation. We discuss the implications of these scenarios for any as yet undiscovered planets in the system, and place the $\eta$ Tel discs in a wider context by comparison to the other $\beta$ Pic moving group A star discs.

\subsection{A young solar system analogue}

The two component model for the $\eta$ Tel disc represents an intriguing analogue to the young solar system. Current theory suggests that the asteroid and Kuiper belts in the Solar System were originally much more massive than they are today and that much of the mass depletion occurred during a chaotic period known as the Late Heavy Bombardment several hundreds of Myr after the formation of the Solar System, possibly triggered by the migration of the giant planets (Gomes et al. 2005). According to the Nice model (Tsiganis et al. 2005), which reproduces fairly accurately the current configuration of the Solar Systems giant planets and the Kuiper Belt (Levison et al. 2007), the Kuiper belt was situated between $\sim 15.5-34 \mathrm{AU}$, with the orbits of the outer planets confined to the 5-15 AU region, whereas the asteroid belt was at its current location. Thus in terms of radial location it is tempting to interpret the $\eta$ Tel disc in terms of an analogous asteroid belt (the unresolved component at 3.9 AU) and an analogous Kuiper belt (the resolved component at $24 \mathrm{AU}$ ). It is worth noting that the luminosity of $\eta$ Tel is much higher than the Sun at $\sim 24 L_{\odot}$, and thus the snow line (the location at which ices are expected to form) would be much further from the star. Using a simple $150 \mathrm{~K}$ blackbody approximation the snow line would be expected at $\sim 17 \mathrm{AU}$ around $\eta \mathrm{Tel}$ (compared to $3.5 \mathrm{AU}$ in the Solar System).

The current levels of excess emission arising from the two populations of debris in the Solar System are 2-3 orders of magnitude lower than that observed around $\eta$ Tel, since the fractional luminosity of asteroid belt dust is $L_{\text {dust }} / L_{\star} \sim 10^{-8}-10^{-7}$ (Dermott et al. 2002), and that of Kuiper belt dust is $L_{\text {dust }} / L_{\star} \sim$ $10^{-7}-10^{-6}$ (Stern \& Colwell 1997). However, the luminosities would have been much higher at an age similar to $\eta$ Tel, since 
this would have been before the depletion of the asteroid and Kuiper belts. The original belts in the Nice model had masses of $\sim 1 M_{\oplus}$ and $\sim 35 M_{\oplus}$ respectively (total mass in full size range of bodies), and so would also have had correspondingly higher levels of dust emission. The fractional luminosity of the Kuiper belt was estimated to be around $5 \times 10^{-4}$ during the pre-LHB phase (Wyatt 2008; Booth et al., in prep.), which is comparable to that of the resolved component of the $\eta$ Tel disc.

\subsection{Origin of the unresolved component}

The fractional luminosity of the pre-LHB asteroid belt would also have been considerably higher, since $1 M_{\oplus}$ of material at $\sim 3$ AU distributed in a collisional cascade size distribution from $2000 \mathrm{~km}$ asteroids down to $0.8 \mu \mathrm{m}$ dust blown out by radiation pressure would have resulted in a fractional luminosity of $\sim 10^{-3}$. However, collisional processing would ensure that such a high mass would not be long-lived, and Wyatt et al. (2007b) showed how there is a maximum fractional luminosity for planetesimal belts of a given age and radius. For a belt at 3.9 AU around $\eta$ Tel that has been undergoing catastrophic disruption for $12 \mathrm{Myr}$ this maximum fractional luminosity is $f_{\max }=4 \times 10^{-6}$. Although the current fractional luminosity is $30-40$ times higher than this maximum value, this does not rule out the possibility that the unresolved component arises from the steady state collisional destruction of an asteroid belt for two reasons. First, the model requires $f_{\text {obs }} / f_{\max } \gg 100$ times higher to indicate a discrepancy given the various uncertainties in the model parameters including assumptions about the maximum planetesimal size, planetesimal strength and orbital eccentricities of around 0.05 used to determine $f_{\max }$ (see also Löhne et al. 2008). Second, it is possible that collisions in the asteroid belt only became destructive after the protoplanetary disc dispersed (after which time eccentricities can increase due to reduced dampening), and so more recently than 12 Myr thus increasing $f_{\text {max }}$.

However, another possible interpretation of emission at a few AU is that it arises in the late stages of the formation of terrestrial planets (e.g., Rhee et al. 2007; Meyer et al. 2008). High levels of hot emission are expected in the planet formation models of Kenyon \& Bromley (2004) once the planetesimal discs are stirred by the formation of Pluto-sized objects. As such objects form within $10^{5}$ yrs at $4 \mathrm{AU}$ in a quiescent disc with the same density as the minimum mass solar nebula (MMSN) (Kenyon \& Bromley 2005), stirring of the disc by a newly formed Pluto does not seem likely to explain the hotter dust emission. However, evidence from the Solar System suggests massive collisions between terrestrial planet cores could occur quite frequently in the first $<100$ Myr (e.g. formation of the Moon, Canup 2004), and models indicate that the final stage of terrestrial planet formation is characterised by such collisions (e.g., Kokubo et al. 2006). Thus the hot emission around $\eta$ Tel could be evidence of stochastic collisions between large rocky cores.

The unresolved component does not represent a population of PR-dragged particles from the resolved ring. Consideration of the collisional and PR-drag timescales for particles from the resolved ring $\left(t_{\text {coll }} / t_{\mathrm{PR}} \approx 0.05\right.$ for a belt at $3.9 \mathrm{AU}$; see Wyatt 2005 , for equations) indicates that any grains susceptible to PR-drag released by collisions in the outer belt are likely to undergo further collisions before PR-drag can affect their orbits to move a significant dust population to the radius of the unresolved component. Furthermore, were PR-drag a dominant process in this disc system, we would expect a dust distribution with a constant surface density (Wyatt 2005), not the two separate populations observed in the Qa images.
It is not possible to distinguish between the asteroid belt and protoplanet collision possibilities at this stage, although detailed spectral modelling may be able to determine the dust composition and so give clues as to the nature of the parent body (e.g., Lisse et al. 2007). Resolving the disc could also aid in its interpretation, although the small spatial scale of this population would make this a challenge. To date only the asteroid belt analogue around $\zeta$ Lep has been resolved at a similar offset from the host star (Moerchen et al. 2007b).

\subsection{Origin of the resolved component}

Although the suggested location and fractional luminosity of the resolved component around $\eta$ Tel compare well with the early Kuiper belt, this does not require the two systems to have undergone a similar evolution and to have similar planetary systems. An alternative scenario for the evolution of the $\eta$ Tel system is given by the self-stirred planet formation models of Kenyon \& Bromley (2004) which may also explain the delay in the onset of $24 \mu \mathrm{m}$ emission peak until 10-15 Myr (Currie et al. 2007). In such models planet formation within an extended planetesimal belt results in a bright ring of emission at the radius where Pluto-sized objects have recently formed. The timescale for the formation of Plutos, $600\left(\Sigma_{0} / \Sigma_{\mathrm{MMSN}}\right)^{-1} r^{3} \mathrm{yr}$, around $\eta$ Tel suggests that to have reached this stage at $24 \mathrm{AU}$ by $12 \mathrm{Myr}$ requires a disc surface density equivalent to $\sim 0.7 \mathrm{MMSN}$.

To assess the possibility that the bright ring in the $\eta \mathrm{Tel}$ disc is caused by the recent formation of Pluto-sized objects at $24 \mathrm{AU}$, we performed additional modelling of the $18 \mu \mathrm{m}$ image based on the prescription for the evolution of dust surface density given in Wyatt (2008) that provides an empirical fit to the selfstirred models of Kenyon \& Bromley (2005). This model considers an extended planetesimal belt of surface density scaled to the MMSN in which annuli at different radii have a suppressed dust content until the age at which Plutos form at which point their mass is eroded through steady state evolution (Wyatt et al. 2007b). The dust emission is calculated assuming a ratio of dust area to planetesimal mass appropriate for a collisional cascade size distribution, and further assuming black body temperatures and emission properties. To account for possible global deviations from this size distribution or from pure black body grains, an additional scaling factor $\eta$ was applied to the surface brightness of the whole disc. An unresolved disc component was included in addition to the unresolved star. The total flux from the self-stirred disc (as fixed by the disc surface density and scaling factor) and the unresolved component was fixed to $259 \mathrm{mJy}$ from the IRS photometry (see Sect. 4). Thus the model has 2 free parameters: $\Sigma_{0} / \Sigma_{\mathrm{MMSN}}$, and $\eta$, with $F_{\text {unres }}$ fixed in response to the level of flux in the self-stirred disc.

As expected from the simple calculation above, the best fitting model has a surface density of 0.7 times MMSN, leading to a ring at $\sim 24 \mathrm{AU}$, with $F_{\text {unres }}=216 \mathrm{mJy}$ (similar to the model of Sect. 4.2) and $\eta=0.22$. The low value of $\eta$ could arise because the grains are cooler than black body, since the ratio of $B_{v}(105 \mathrm{~K}) / B_{v}(123 \mathrm{~K})$ is $\sim 0.3$ at $18 \mu \mathrm{m}$. The $\chi^{2}$ for this model is 58 with a reduced $\chi^{2}$ of $\chi_{\min }^{2}=1.21$, slightly higher than that of the 2 parameter model $\left(\chi_{\min }^{2}=1.13\right)$ in Sect. 4.2. The reduced $\chi^{2}$ of the image (taken as described in Sect. 4.2) was 1.15, better than the two parameter model which had $\chi_{\min }^{2}=1.3$. The BIC for this model is 32 , which is indicative of the almost equally good fit of the two models. The BIC values show there is no evidence against either the simple ring plus unresolved flux model (Sect. 4.2) or the self-stirred model. The residuals for the best 


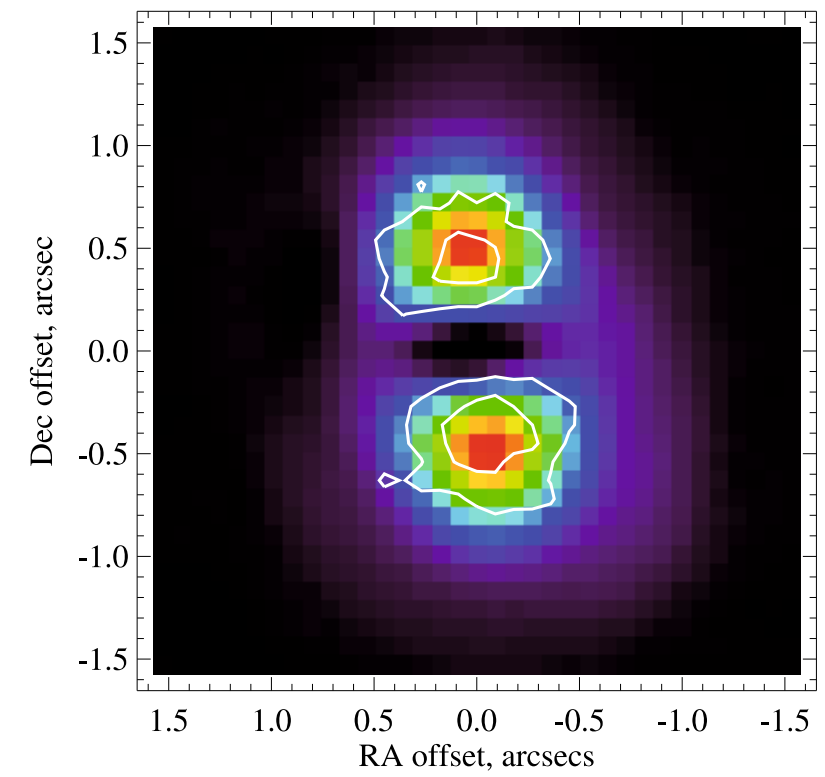

Fig. 5. The self stirred model of the resolved disc component as described in Sect. 5.3 after the same PSF subtraction as applied to the observations (Fig. 1 Qa band residual image) and 2 parameter model, shown in Fig. 3. The scaling in this image is the same as for Fig. 1 top left. Contours are from the observed residuals image, at levels of $1 / 3$ and $2 / 3$ of the peak, as for Fig. 3 .

fitting self-stirred model are plotted in Fig. 5. Multi-wavelength imaging may help break this degeneracy. The self-stirred model requires an extended disc with a brightness peak at the location of recent Pluto formation. If a larger disc location were observed in longer wavelength imaging this could be evidence in favour of the self-stirred interpretation, whereas in the solar system analogy we may expect to see the disc confined to a narrow radial range. Alternatively searching for planets in the system could further test these models.

In other words the structure of the disc is entirely consistent with a self-stirred extended planetesimal belt in which the inner region has been cleared through collisional erosion following the formation of Pluto-sized objects. One caveat is that an additional unresolved component still has to be invoked. To be consistent such a component could not originate in an asteroid belt (unless this had significantly different material properties to the outer regions), and further work is needed to assess the duration and radial location of any $18 \mu \mathrm{m}$ emission expected from the final collisional phase of terrestrial planet formation.

\subsection{Implications for the planetary system}

Taking the solar system analogy to its extreme would suggest that the gap in the dust distribution seen around $\eta$ Tel at 5-20 AU is caused by the existence of giant planets distributed in this region. Like the Solar System, the planetesimal belt at 3.9 AU could represent a region where terrestrial planet formation was disrupted by the influence of a gas giant planet at 5-6 AU, and the inner edge of the belt at 24 AU could demark the outer edge of the planetary system.

The inner edge of the the hot dust could also be evidence of terrestrial planets at $<3 \mathrm{AU}$, as the hot dust at $1 \mathrm{AU}$ around HD 69830 (Beichman et al. 2005; Lisse et al. 2007) lies just outside 3 Neptune mass planets, the outermost of which is at $0.63 \mathrm{AU}$ (Lovis et al. 2006) (although the location of the disc has not yet been confirmed through resolved imaging). Also, although no planets have been detected around zeta Lep, the restricted spatial extent of its asteroid belt (Moerchen et al. 2007b) is suggested to be possible evidence of perturbations of planetary bodies.

Implicit in the self-stirring interpretation is the formation of planets at least the size of Pluto in the inner regions. Such objects would presumably have grown through mutual collisions to larger sizes, but it is not required that they also evolved into gas giant planets, which would depend on whether they reached $\sim 10 M_{\oplus}$ before the gas disc dissipated (e.g. Lissauer 1993).

A further possibility which is not discussed here is that this disc is a remnant of the protoplanetary disc, and that the dust is shepherded into a ring through the action of gas drag forces or instabilities (Takeuchi \& Artymowicz 2001; Klahr \& Lin 2005; Besla \& Wu 2007). In this case it would not be necessary for any planets to be present. A massive gas disc around a star of this age would be highly unusual, as most gas discs are believed to dissipate in $<10 \mathrm{Myr}$ (e.g. Hollenbach et al. 2005), although gas has been detected around 4 A stars ( $\beta$ Pic, Brandeker et al. 2004; HD 141596, Jonkheid et al. 2006; 49 Ceti, Dent et al. 2005; and HD 32297, Redfield 2007). To assess this possibility knowledge of the gas distribution is vitally important, yet gas has yet to be detected around $\eta$ Tel.

\subsection{Comparison with A star discs in $\beta$ Pic moving group}

Of the 5 A stars in the $\beta$ Pic moving group, 3 exhibit excess midIR emission from a debris disc ( $\beta$ Pic, $\eta$ Tel and HD 172555). The derived (or directly measured) structures of these 3 discs display significant diversity. $\beta$ Pic, an A6V star, has a well known highly extended disc with dust imaged from $10 \mathrm{~s}$ of $\mathrm{AU}$ to an outer extent of $\sim 1500$ AU (Telesco et al. 2005; Larwood \& Kalas 2001). Since dust at the outer edge is thought to be created in planetesimal belts much closer to the star (Augereau et al. 2001), it is probably most instructive to consider this disc as an extended planetesimal belt out to $100 \mathrm{AU}$ with a broad peak in surface density at around 70 AU (Telesco et al. 2005). HD 172555 on the other hand has all of its emission much closer to the star. Its emission spectrum indicates the presence of silicate grains at $520 \mathrm{~K}$ and a blackbody component at $200 \mathrm{~K}$ implying a radial offset of 0.8-6 AU (Chen et al. 2006; Rebull et al. 2008). The $\sim 24 \mathrm{AU}$ outer belt of the $\eta$ Tel debris lies intermediate between these two extremes. These discs thus represent the diversity that is seen amongst debris discs at the age when emission is seen to peak (Currie et al. 2007).

Within the context of the self-stirring model this diversity would be expected to arise primarily from different initial disc masses. While $\eta \mathrm{Tel}$ is the product of a $0.7 \mathrm{MMSN}$ disc, the protoplanetary disc of HD 172555 (and of the two A stars which are not detected) is presumably of lower density, and that of $\beta$ Pic is of higher density to allow Plutos to form out to $70 \mathrm{AU}$ by $12 \mathrm{Myr}$. It is also worth noting that the beta Pic disc shows an asymmetry that may be the result of a catastrophic collision at $\sim 50 \mathrm{AU}$, such as might be expected from the self-stirring models at the epoch of Pluto-formation. Such a diversity of protoplanetary disc masses is expected (e.g., Andrews \& Williams 2005), but the small number of stars in this sample does not permit any definite conclusions to be drawn. In the young Solar System interpretation, the diversity in these discs could instead be due to the diversity in planetary system architecture across the three stars, which in addition to a dependence on initial disc mass (Wyatt et al. 2007a) could also have a significant stochastic component (e.g. Quintana \& Lissauer 2006, find small changes in the location of planetary embryos has a great impact on the final system 
architecture). Furthermore the origin of the hot emission, and its diversity, remains a puzzle, and it is not clear whether the presence, or lack thereof, of asteroid belts and ongoing terrestrial planet formation should be a monotonic function of the initial disc mass (see e.g. Su et al. 2008, who find from a sample of middle-aged discs around A0-A3V stars that factors beyond variation in initial disc mass are needed to explain the variation in observed disc structure).

\section{Conclusions}

We have presented Si-5 and Qa band Gemini T-ReCS imaging of the $12 \mathrm{Myr}$ old A star $\eta$ Tel. The emission at $18.3 \mu \mathrm{m}$ is shown to be significantly extended compared with a point source. This image represents the first resolution of dust emission around this star. These observations confirm the interpretation of the SED as a debris disc comprised of two populations: one component that is resolved at $24 \mathrm{AU}$, the other that is unresolved with a temperature consistent with dust at 3.9 AU.

Two interpretations are proposed for the origin of the architecture of these debris belts. (i) The system is a close analogue to the Solar System at a similar age, both in terms of the radial location of the debris and the level of emission; i.e., these belts represent analogous asteroid and Kuiper belts. (ii) Alternatively this system is still undergoing planet formation, and the $24 \mathrm{AU}$ ring is the location where Pluto-sized objects recently formed; the 3.9 AU ring could be the product of recent collisions between Mars-sized bodies in the final accumulation phases of terrestrial planets. This implies that this system evolved from a 0.7 MMSN protoplanetary disc.

These possibilities can be further tested by probing the radial structure of the disc through multiple wavelength imaging, and by searching for planets both through direct methods, or through looking for evidence for them in the disc structure. Thus $\eta$ Tel is a valuable source for testing planet formation theories, not least since it may be an example of a system where planet formation is ongoing.

Acknowledgements. R.S. is grateful for the support of a Fellowship of the Royal Commission for the Exhibition of 1851. L.J.C. is grateful for the support of an STFC studentship. M.C.W. is grateful for the support of a Royal Society University Research Fellowship. M.M. acknowledges support from JPL funded by NASA through the Michelson Fellowship Program. JPL is managed for NASA by the California Institute of Technology. The authors would like to thank Christine Chen for providing the Spitzer IRS spectrum of $\eta$ Tel. Based on observations obtained at the Gemini Observatory, which is operated by the Association of Universities for Research in Astronomy, Inc., under a cooperative agreement with the NSF on behalf of the Gemini partnership: the National Science Foundation (United States), the Science and Technology Facilities Council (United Kingdom), the National Research Council (Canada), CONICYT (Chile), the Australian Research Council (Australia), Ministério da Ciência e Tecnologia (Brazil) and SECYT (Argentina).

\section{References}

Andrews, S. M., \& Williams, J. P. 2005, ApJ, 631, 1134

Augereau, J. C., Lagrange, A. M., Mouillet, D., Papaloizou, J. C. B., \& Grorod, P. A. 1999 , A\&A, 348, 557
Augereau, J. C., Nelson, R. P., Lagrange, A. M., Papaloizou, J. C. B., \& Mouillet, D. 2001, A\&A, 370, 447

Aumann, H. H., \& Probst, R. G. 1991, ApJ, 368, 264

Backman, D. E., \& Paresce, F. 1993, in Protostars and Planets III, ed. E. H. Levy \& J. I. Lunine (Tucson: University of Arizona Press), 1253

Beichman, C. A., Bryden, G., Gautier, T. N., et al. 2005, ApJ, 626, 1061

Besla, G., \& Wu, Y. 2007, ApJ, 655, 528

Bohren, C. F., \& Huffman, D. R. 1983, Absorption and scattering of light by small particles (New York: Wiley)

Brandeker, A., Liseau, R., Olofsson, G., \& Fridlund, M. 2004, A\&A, 413, 681 Canup, R. M. 2004, ARA\&A, 42, 441

Chen, C. H., Sargent, B. A., Bohac, C., et al. 2006, ApJS, 166, 351

Cohen, M., Walker, R. G., Carter, B., et al. 1999, AJ, 117, 1864

Currie, T., Kenyon, S., Balog, Z., et al. 2007, ArXiv e-prints: 0709.2510

Dent, W. R. F., Greaves, J. S., \& Coulson, I. M. 2005, MNRAS, 359, 663

Dermott, S. F., Durda, D. D., Grogan, K., \& Kehoe, T. J. J. 2002, Asteroids III, 423

Dohnanyi, J. 1969, J. Geophys. Res., 74, 2531

Fisher, R. S., Telesco, C. M., Piña, R. K., Knacke, R. F., \& Wyatt, M. C. 2000, ApJ, 532, L141

Gomes, R., Levison, H. F., Tsiganis, K., \& Morbidelli, A. 2005, Nature, 435, 466 Hauschildt, P. H., Allard, F., \& Baron, E. 1999, ApJ, 512, 377

Hollenbach, D., Gorti, U., Meyer, M., et al. 2005, ApJ, 631, 1180

Jayawardhana, R., Fisher, S., Hartmann, L., et al. 1998, ApJ, 503, L79

Jonkheid, B., Kamp, I., Augereau, J.-C., \& van Dishoeck, E. F. 2006, A\&A, 453, 163

Kenyon, S. J., \& Bromley, B. C. 2002, ApJ, 577, L35

Kenyon, S. J., \& Bromley, B. C. 2004, AJ, 127, 513

Kenyon, S. J., \& Bromley, B. C. 2005, AJ, 130, 269

Klahr, H., \& Lin, D. N. C. 2005, ApJ, 632, 1113

Kokubo, E., Kominami, J., \& Ida, S. 2006, ApJ, 642, 1131

Larwood, J. D., \& Kalas, P. G. 2001, MNRAS, 323, 402

Levison, H. F., Morbidelli, A., Van Laerhoven, C., Gomes, R., \& Tsiganis, K. 2007, ArXiv e-prints, 712

Li, A., \& Greenberg, J. M. 1998, A\&A, 331, 291

Liddle, A. R. 2004, MNRAS, 351, L49

Lissauer, J. J. 1993, ARA\&A, 31, 129

Lisse, C. M., Beichman, C. A., Bryden, G., \& Wyatt, M. C. 2007, ApJ, 658, 584

Löhne, T., Krivov, A. V., \& Rodmann, J. 2008, ApJ, 673, 1123

Lovis, C., Mayor, M., Pepe, F., et al. 2006, Nature, 441, 305

Lowrance, P. J., Schneider, G., Kirkpatrick, J. D., et al. 2000, ApJ, 541, 390

Meyer, M. R., Carpenter, J. M., Mamajek, E. E., et al. 2008, ApJ, 673, L181

Moerchen, M. M., Telesco, C. M., De Buizer, J. M., Packham, C., \& Radomski, J. T. 2007a, ApJ, 666, L109

Moerchen, M. M., Telesco, C. M., Packham, C., \& Kehoe, T. J. J. 2007b, ApJ, 655, L109

Quintana, E. V., \& Lissauer, J. J. 2006, Icarus, 185, 1

Rebull, L. M., Stapelfeldt, K. R., Werner, M. W., et al. 2008, ArXiv e-prints, 803 Redfield, S. 2007, ApJ, 656, L97

Rhee, J. H., Song, I., Zuckerman, B., \& McElwain, M. 2007, ApJ, 660, 1556

Schwarz, G. 1978, Ann. Statist., 5, 461

Smith, R., Wyatt, M. C., \& Dent, W. R. F. 2008, A\&A, 485, 897

Stern, S. A., \& Colwell, J. E. 1997, AJ, 114, 841

Su, K. Y. L., Rieke, G. H., Stapelfeldt, K. R., et al. 2008, ApJ, 679, L125

Takeuchi, T., \& Artymowicz, P. 2001, ApJ, 557, 990

Telesco, C. M., Fisher, R. S., Piña, R. K., et al. 2000, ApJ, 530, 329

Telesco, C. M., Fisher, R. S., Wyatt, M. C., et al. 2005, Nature, 433, 133

Tsiganis, K., Gomes, R., Morbidelli, A., \& Levison, H. F. 2005, Nature, 435, 459

Wahhaj, Z., Koerner, D. W., Backman, D. E., et al. 2005, ApJ, 618, 385

Wahhaj, Z., Koerner, D. W., \& Sargent, A. I. 2007, ApJ, 661, 368

Wyatt, M. C. 2005, A\&A, 433, 1007

Wyatt, M. C. 2008, ARA\&A, 46, 339

Wyatt, M. C., Dermott, S. F., Telesco, C. M., et al. 1999, ApJ, 527, 918

Wyatt, M. C., Clarke, C. J., \& Greaves, J. S. 2007a, MNRAS, 380, 1737

Wyatt, M. C., Smith, R., Greaves, J. S., et al. 2007b, ApJ, 658, 569

Zuckerman, B., Song, I., Bessell, M. S., \& Webb, R. A. 2001, ApJ, 562, L87 